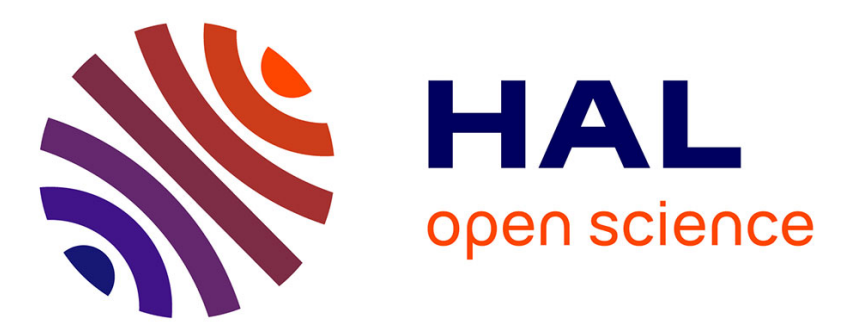

\title{
Comparative static curing versus dynamic curing on tablet coating structures
}

Claire Gendre, Muriel Genty, Barbara Fayard, Ali Tfayli, Mathieu Boiret, Olivier Lecoq, Michel Baron, Pierre Chaminade, Jean Manuel Pean

\section{To cite this version:}

Claire Gendre, Muriel Genty, Barbara Fayard, Ali Tfayli, Mathieu Boiret, et al.. Comparative static curing versus dynamic curing on tablet coating structures. International Journal of Pharmaceutics, 2013, 453 (2), pp.448-453. 10.1016/j.ijpharm.2013.06.008 . hal-01631583

\section{HAL Id: hal-01631583 https://hal.science/hal-01631583}

Submitted on 6 Nov 2019

HAL is a multi-disciplinary open access archive for the deposit and dissemination of scientific research documents, whether they are published or not. The documents may come from teaching and research institutions in France or abroad, or from public or private research centers.
L'archive ouverte pluridisciplinaire HAL, est destinée au dépôt et à la diffusion de documents scientifiques de niveau recherche, publiés ou non, émanant des établissements d'enseignement et de recherche français ou étrangers, des laboratoires publics ou privés. 


\title{
Comparative static curing versus dynamic curing on tablet coating structures
}

\author{
Claire Gendre ${ }^{\mathrm{a}, \mathrm{b}, \mathrm{c}, *}$, Muriel Genty ${ }^{\mathrm{c}}$, Barbara Fayard ${ }^{\mathrm{d}}$, Ali Tfayli ${ }^{\mathrm{a}}$, Mathieu Boiret ${ }^{\mathrm{c}}$, \\ Olivier Lecoq $^{\mathrm{b}}$, Michel Baron ${ }^{\mathrm{b}}$, Pierre Chaminade ${ }^{\mathrm{a}}$, Jean Manuel Péan ${ }^{\mathrm{c}}$ \\ a Univ Paris-Sud, EA 4041, Groupe de Chimie Analytique de Paris-Sud, IFR 141-IPSIT, Faculty of Pharmacy, 92296 Châtenay-Malabry, France \\ ${ }^{\mathrm{b}}$ Université de Toulouse, Mines Albi, CNRS UMR 5302, Centre RAPSODEE, Campus Jarlard, F-81013 Albi CT cedex 09, France \\ ${ }^{\mathrm{c}}$ Technologie Servier, 27 rue Eugène Vignat, 45000 Orléans, France \\ d Novitom, 155-157 cours berriat, 38000 Grenoble, France
}

Keywords:

Curing

Film-coating structures

Aqueous ethylcellulose dispersion

Near infrared spectroscopy

X-ray micro-computed tomography

X-ray microdiffraction

\begin{abstract}
A B S T R A C T
Curing is generally required to stabilize film coating from aqueous polymer dispersion. This post-coating drying step is traditionally carried out in static conditions, requiring the transfer of solid dosage forms to an oven. But, curing operation performed directly inside the coating equipment stands for an attractive industrial application. Recently, the use of various advanced physico-chemical characterization techniques i.e., X-ray micro-computed tomography, vibrational spectroscopies (near infrared and Raman) and X-ray microdiffraction, allowed new insights into the film-coating structures of dynamically cured tablets. Dynamic curing end-point was efficiently determined after $4 \mathrm{~h}$. The aim of the present work was to elucidate the influence of curing conditions on film-coating structures. Results demonstrated that $24 \mathrm{~h}$ of static curing and $4 \mathrm{~h}$ of dynamic curing, both performed at $60^{\circ} \mathrm{C}$ and ambient relative humidity, led to similar coating layers in terms of drug release properties, porosity, water content, structural rearrangement of polymer chains and crystalline distribution. Furthermore, X-ray microdiffraction measurements pointed out different crystalline coating compositions depending on sample storage time. An aging mechanism might have occur during storage, resulting in the crystallization and the upward migration of cetyl alcohol, coupled to the downward migration of crystalline sodium lauryl sulfate within the coating layer. Interestingly, this new study clearly provided further knowledge into film-coating structures after a curing step and confirmed that curing operation could be performed in dynamic conditions.
\end{abstract}

\section{Introduction}

Aqueous-based coatings of polymer dispersions have been developed as an alternative to organic solutions presenting environmental and safety concerns. Moreover, aqueous functional coatings are often used for controlled drug release applications (Porter et al., 2009). However, mechanisms of film formation differ for solutions and dispersions (Felton, 2013). Aqueous polymer dispersions generally require a post-coating drying step, known as curing, to achieve complete coalescence and provide stable drug release profiles (Bodmeier and Paeratakul, 1994; Siepmann et al., 2007). Curing is traditionally carried out in static conditions, in an oven, but can preferably be performed inside the coating equipment, under dynamic conditions. The additional transfer step can

\footnotetext{
* Corresponding author at: Technologie Servier, 27 rue Eugène Vignat, 45000 Orléans, France. Tel.: +33 02382380 00; fax: +330238238201.

E-mail address: claire.gendre@gmail.com (C. Gendre).
}

thus be eliminated, reducing considerably curing time (Baer et al., 2008; Muschert et al., 2011). Therefore, dynamic curing represents a great interest in an industrial context.

Furthermore, several critical factors such as relative humidity $(\mathrm{RH})$, temperature and time during the curing operation can have a significant effect on solid dosage form properties (Wesseling and Bodmeier, 1999; Liu and Williams, 2002; Körber et al., 2010).

In a recent study, a new insight into tablet coating structures along a dynamic curing process, inside a pan coater at $60^{\circ} \mathrm{C}$ and ambient relative humidity, was provided (Gendre et al., 2012). Various techniques, such as X-ray micro-computed tomography $(\mathrm{X} \mu \mathrm{CT}), \mathrm{X}$-ray microdiffraction, as well as near infrared (NIR) and Raman spectroscopies, were jointly used as non-destructive techniques to allow advanced physico-chemical characterization of solid dosage forms. Dynamic curing end-point was accurately determined after $4 \mathrm{~h}$. It was demonstrated that a stabilized state was reached in terms of internal coating porosity, NIR and Raman spectral information and crystalline distribution. These results were subsequently confirmed by dissolution stability studies. 
Table 1

Coating composition for $1.0 \mathrm{~kg}$ of core tablets.

\begin{tabular}{ll}
\hline Materials & Suspension $(\mathrm{g})$ \\
\hline Aquacoat ECD 30 & 450.0 \\
Kollicoat IR $^{\circledR \mathrm{b}}$ & 15.0 \\
Citroflex 2 & 33.8 \\
Purified water & 420.2 \\
\hline${ }^{\text {a }}$ Aqueous ethylcellulose dispersion (FMC Biopolymer, Philadelphia, USA). \\
b Poly(vinyl alcohol)-poly(ethylene glycol) graft copolymer (BASF, Ludwigshafen, \\
Germany).
\end{tabular}

However, these findings were not completely correlated to drug release properties as similar dissolution profiles were reached after only 30 min of dynamic curing.

As previously mentioned, static curing is currently considered as standard curing. For the presently used coating formulation, a functional polymer combination of ethylcellulose/poly(vinyl alcohol)-poly(ethylene glycol) graft copolymer, our objectives were to compare tablet-coating structures subjected to either $24 \mathrm{~h}$ of static curing or $4 \mathrm{~h}$ of dynamic curing and to confirm the efficiency of the dynamic curing operation. In addition to the standard dissolution method, deeper characterization of film-coating layers was provided using the aforementioned techniques to determine the influence of curing conditions.

\section{Materials and methods}

\subsection{Materials and preparation of cured coated tablets}

Materials and method for the preparation of coated tablets were previously described in detail (Gendre et al., 2012). Hydrophilic matrix tablets with biconvex shape ( $8 \mathrm{~mm}$ diameter, average weight: $200 \mathrm{mg}$ ), including hypromellose and calcium hydrogenophosphate as main excipients and a freely soluble drug substance, were supplied by Les Laboratoires Servier Industrie (Gidy, France). Coating composition is provided in Table 1. Film coating was performed in a partially side-vented pan coater at $4.0 \mathrm{~kg}$ scale (Driacoater $500^{\circledR}$, Driam, Eriskirch, Germany). Process parameters are listed in Table 2.

Coating was stopped when the theoretically applied amount of coating per tablet was $24.5 \mathrm{mg}$ corresponding to a weight gain of $12.5 \%$ of the core tablet weight.

Tablets were then cured either under static or under dynamic conditions. Approximately 100 tablets were collected at the end of the coating operation and subjected to traditional oven curing $\left(24 \mathrm{~h}\right.$ at $60^{\circ} \mathrm{C}$ and ambient relative humidity; vented oven, Lequeux, France). Coated tablets remaining inside the pan coater were dynamically cured over a $4 \mathrm{~h}$ period $(10 \mathrm{rpm}$; inlet temperature of $65^{\circ} \mathrm{C}$; product temperature of $60^{\circ} \mathrm{C}$; ambient relative humidity). Approximately 50 tablets were collected at regular time intervals throughout the dynamic curing process (every $30 \mathrm{~min}$ for the first hour, then every hour until $6 \mathrm{~h}$ ).

All experiments were carried out in temperate $\left(20^{\circ} \mathrm{C}\right)$ and controlled relative humidity ( $50 \% \mathrm{RH}$ ) environment.

Table 2

Coating process parameters.

\begin{tabular}{ll}
\hline Process parameters & Values \\
\hline Spray nozzle diameter & $0.8 \mathrm{~mm}$ \\
Spray rate & $20 \mathrm{~g} / \mathrm{min}$ \\
Spray pressure & $2.4 \mathrm{bars}$ \\
Pan speed & $20 \mathrm{rpm}$ \\
Inlet temperature & $60^{\circ} \mathrm{C}$ \\
Inlet air humidity & $26 \%$ \\
Product temperature & $36^{\circ} \mathrm{C}$ \\
\hline
\end{tabular}

\subsection{Curing characterization}

Various techniques, i.e., dissolution testing, X-ray microcomputed tomography, X-ray microdiffraction, near infrared and Raman spectroscopies were recently used to characterize dynamically cured tablets (Gendre et al., 2012). These same techniques were also used in this study to determine the influence of curing conditions on film-coating structures. A brief description of their set-up parameters is given below.

\subsection{Dissolution studies}

In vitro drug release from cured tablets was evaluated using the USP apparatus 2 dissolution system (AT7 Smart Off-line, Sotax, Allschiwtz, Switzerland), in $0.05 \mathrm{M}$ phosphate buffer at $\mathrm{pH} 6.8$ $\left(1000 \mathrm{~mL} ; 37^{\circ} \mathrm{C}\right.$; $50 \mathrm{rpm}$; three tablets analyzed per curing condition). $10 \mathrm{ml}$ samples were regularly withdrawn over a $16-\mathrm{h}$ period and analyzed by spectrophotometry $(\lambda=230 \mathrm{~nm}$, Agilent 8453 UV-vis spectroscopy system, Hewlett Packard, Waldbronn, Germany).

Storage stability of cured tablets, stored in closed polypropylene vials, was assessed under both ambient conditions $\left(25^{\circ} \mathrm{C}\right.$ and $60 \% \mathrm{RH} ; 18$ months storage) and accelerated conditions ( $40^{\circ} \mathrm{C}$ and $75 \% \mathrm{RH} ; 6$ months storage). Dissolution profiles were then obtained according to the previously described method.

\subsection{X-ray micro-computed tomography $(X \mu C T)$ measurements}

High-resolution X-ray microtomography acquisitions were performed on ID19 beamline at the European Synchrotron Radiation Facility (Grenoble, France). Set-up parameters were as follows: $1200 \mathrm{X}$-ray radiographies per sample; sample rotation over a range of $180^{\circ}$; spatial resolution of $0.28 \mu \mathrm{m}$; sample-detector distance of $12 \mathrm{~mm}$; energy of incident X-ray parallel beam of $17.6 \mathrm{keV}$; beam dimensions of $600 \mu \mathrm{m}$ (wide) $\times 300 \mu \mathrm{m}$ (high); one tablet analyzed per curing condition. 3D images were reconstructed using a standard filtered back projection algorithm, corresponding to a cylindrical reconstructed volume of $600 \mu \mathrm{m}$ in diameter and $300 \mu \mathrm{m}$ in height. Quantitative analysis of coating porosity, expressed as the ratio (volume of pores) to (total volume), was performed from a basic segmentation of the reconstructed volume using threshold adjustment and manual refinement (selected volume of $2048 \times 2048 \times 200$ pixels $^{3}$, VGStudio MAX 2.1 software). The 200 pixels corresponded to a zone chosen in the middle of the coating in order to avoid artifacts from the surface or from the tablet core interface.

\subsection{Near infrared and Raman measurements}

A BUCHI NIRFlex N500 spectrometer (BUCHI Labortechnik AG, Switzerland) was used for NIR spectral acquisitions (diffuse reflectance mode; illumination spot diameter of $4 \mathrm{~mm}$; $\Delta \lambda=12,200-4000 \mathrm{~cm}^{-1}$; spectral resolution of $16 \mathrm{~cm}^{-1} ; 128$ scans accumulated; fifteen tablets analyzed per curing condition).

A HR Labram microspectrometer (Horiba Jobin Yvon, France) was used to perform Raman spectral acquisitions $(785 \mathrm{~nm}$ generating approximately $50 \mathrm{~mW}$ on the sample; CCD detector $\left(1024 \times 256\right.$ pixels $\left.{ }^{2}\right)$; spectral resolution of $2 \mathrm{~cm}^{-1}$; $\Delta \lambda=500-1800 \mathrm{~cm}^{-1}$; total laser light exposure time of $60 \mathrm{~s}$ per collected spectrum; three tablets analyzed per curing condition with 3 acquisitions per sample at different positions on the surface).

All Raman and NIR data were exported from Labspec5 software and BUCHI NIRCal ${ }^{\circledR}$ software, respectively, and analyzed using Matlab ${ }^{\circledR} 7.8$, R2009a software (The MathWorks Inc., Natick, USA) and PLS Toolbox 5.8.3 (Eigenvector Research Inc., Wenatchee, 


\section{4 h static curing}
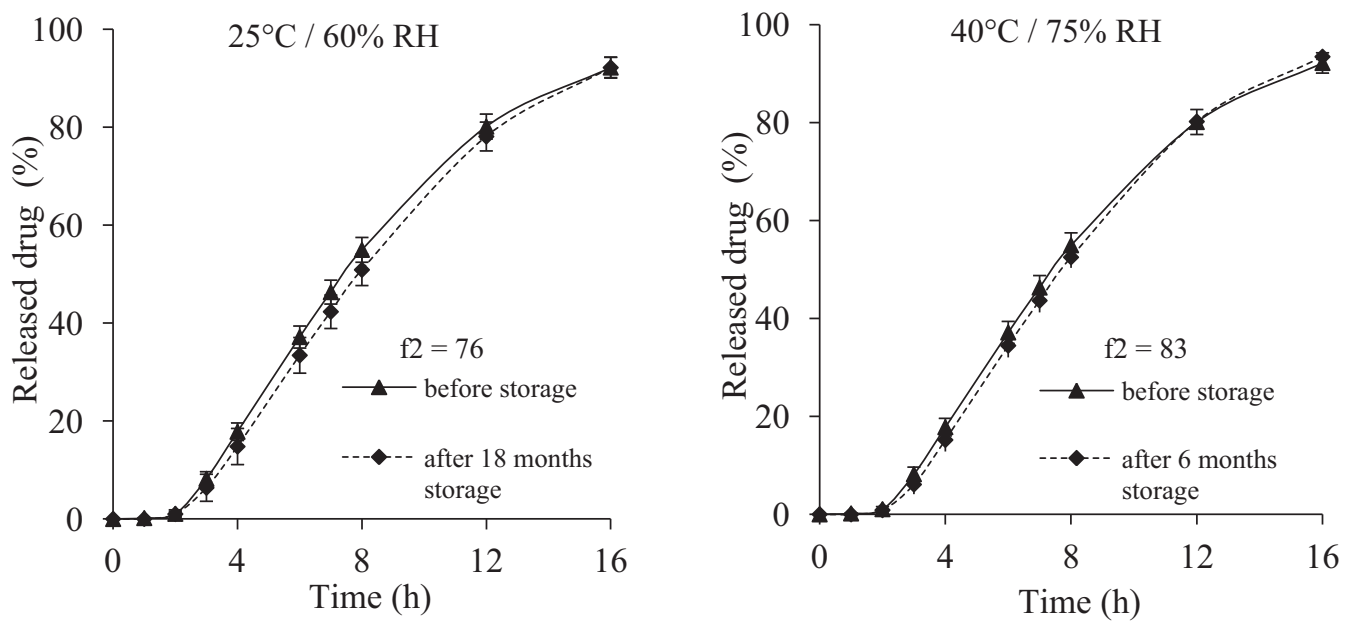

Fig. 1. Storage stability of coated tablets cured for $24 \mathrm{~h}$ in static conditions, stored during 18 months under ambient conditions (left) and 6 months under stress conditions (right) (coating level: 10\% ethylcellulose: PVA-PEG graft copolymer 90:10).

USA). A multivariate qualitative analysis using Principal Component Analysis (PCA) was applied on the pre-processed NIR spectra (second derivative and Savitzky-Golay smoothing: filter width of 9 points and a second-order polynomial fitting) (Naes et al., 2002). Raman spectra were pre-processed using baseline correction and normalization based on the highest peak intensity.

\subsection{X-ray microdiffraction measurements}

Experiments were performed at the European Synchrotron Radiation Facility (Grenoble, France) on the ID13 microfocus beamline. Set-up parameters were as follows: beam energy of $12.46 \mathrm{keV}$ $(\lambda=0.995 \AA)$; beam dimensions of $1.5 \times 1.2 \mu \mathrm{m}^{2}$; sample-detector distance of $145.1 \mathrm{~mm}$; one tablet analyzed per curing condition. Using a $\sim 200 \mu \mathrm{m}$ diameter beam-stop, two-dimensional X-ray scattering patterns were recorded from 0.01 to $0.5 \AA^{-1}$ on a homemade FRELON camera $\left(2048 \times 2048\right.$ pixels $^{2}$; pixel size of $50.0 \times 50.0 \mu \mathrm{m}^{2}$ ). The data collection procedure consisted of a series of 100 shots collected along a $200 \mu \mathrm{m}$ long line perpendicular to the coating surface with a $2 \mu \mathrm{m}$ step size. Data were analyzed using the FIT2D software.

\section{Results and discussion}

\subsection{Influence of curing conditions on drug release properties}

The long-term storage stability of film coatings subjected to $4 \mathrm{~h}$ of dynamic curing was recently demonstrated (Gendre et al., 2012). As it can be observed in Fig. 1, stable drug release patterns were also obtained from $24 \mathrm{~h}$ traditionally cured tablets. Clearly, no significant difference was shown after 18 months storage at $25^{\circ} \mathrm{C}$ and $60 \% \mathrm{RH}$ (ambient conditions) and 6 months storage at $40^{\circ} \mathrm{C}$ and $75 \% \mathrm{RH}$ (stress conditions). The values of similarity factor $\mathrm{f} 2$ were greater than 50 , respectively 76 and 83 , ensuring the equivalence of the two dissolution profiles (Moore and Flanner, 1996). Therefore, the high stability of film coatings after a 24 -h period of curing step in static conditions was confirmed.

These results obtained from tablets cured in static conditions during $24 \mathrm{~h}$ were in agreement with Muschert's study (Muschert et al., 2011). For closely similar coating composition but different core composition, the authors reported long-term stable film coatings from solid dosage forms cured in similar conditions $(24 \mathrm{~h}$ in an oven at $60^{\circ} \mathrm{C}$ at ambient relative humidity/storage under ambient conditions for 12 months).

Drug release rates from $24 \mathrm{~h}$ oven cured tablets and from $4 \mathrm{~h}$ dynamically cured tablets were then compared (Fig. 2). Similar dissolution profiles were obtained as highlighted by the similarity factor of 63 . The efficiency of the dynamic curing operation was therefore reinforced. This result represents a great interest in terms of industrial application. Coated tablets requiring an additional curing step can be cured directly inside the coating apparatus rather than transferred to an oven.

Nevertheless, it was previously demonstrated that dissolution testing failed to differentiate the dissolution profiles obtained from tablets cured during dynamic curing times longer than $30 \mathrm{~min}$ (Gendre et al., 2012). The use of advanced physico-chemical characterization techniques such as X $\mu \mathrm{CT}$, NIR and Raman spectroscopies and $\mathrm{X}$-ray microdiffraction provided a better insight into film-coating structures. Indeed, slight variations were successfully detected until a stable state was reached after $4 \mathrm{~h}$ of dynamic curing.

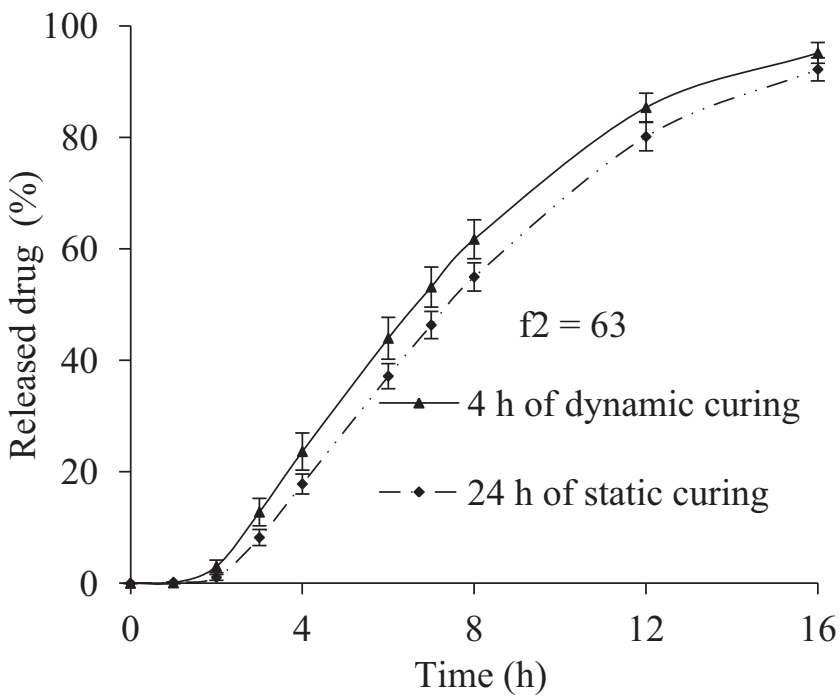

Fig. 2. Influence of curing conditions on drug release properties from coated tablets after $24 \mathrm{~h}$ of static curing and $4 \mathrm{~h}$ of dynamic curing, both carried out at $60^{\circ} \mathrm{C}$ and ambient relative humidity. The results obtained from $4 \mathrm{~h}$ of dynamic curing are reported for comparison from Gendre et al. (2012). 

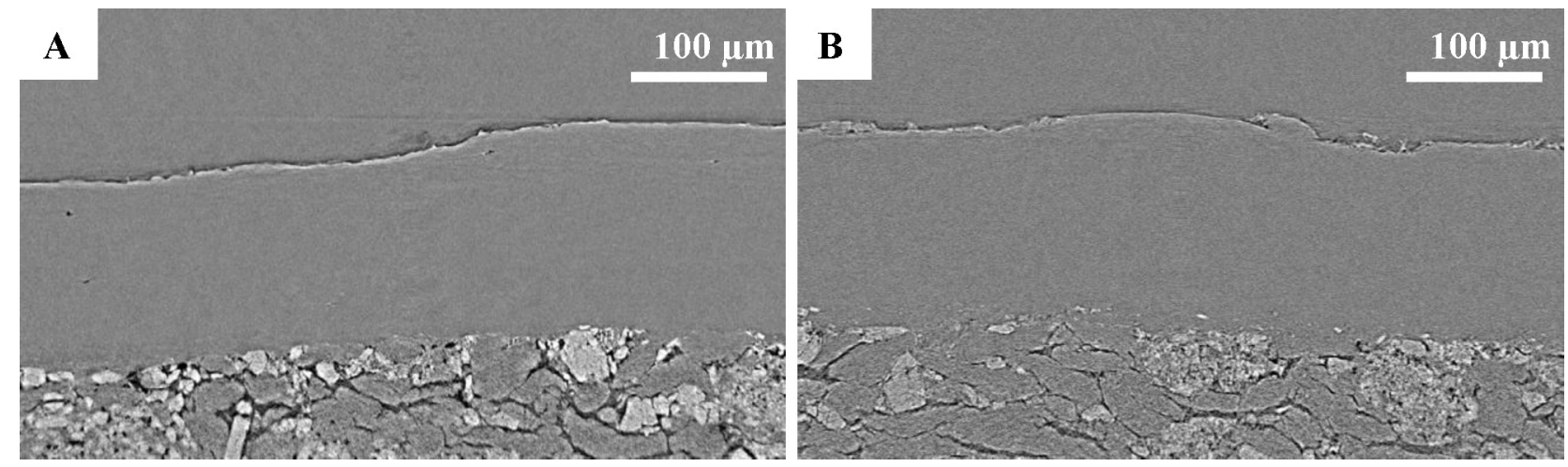

Fig. 3. Cross-section images (A) of $24 \mathrm{~h}$ traditionally oven cured tablet and (B) of $4 \mathrm{~h}$ dynamically cured tablet (image reproduced from Gendre et al., 2012).

In the present work, these techniques were jointly used to elucidate the influence of curing conditions on film-coating structures and to confirm similar dissolution results obtained for static curing and dynamic curing.

\subsection{Comparison of internal coating structures using $X \mu C T$}

$\mathrm{X} \mu \mathrm{CT}$ measurements allow the detection of subtle variations within the internal structure of solid dosage forms by a nondestructive image analysis (Poutiainen et al., 2011; Monkare et al., 2012; Noguchi et al., 2013). The use of this technique recently highlighted the presence of micro pores within the coating layers of cured tablets, which gradually decreased as dynamic curing time increased (Gendre et al., 2012).

The 2D visualizations of the coating structures for tablet cured in static conditions and for tablet dynamically cured during $4 \mathrm{~h}$ are depicted in Fig. 3. A qualitative observation of these two $\mathrm{X} \mu \mathrm{CT}$ images, at this magnification scale, revealed similar coating structures characterized by low internal porosity. This result was confirmed by a quantitative volume analysis, showing porosity of $0.04 \% \mathrm{v} / \mathrm{v}$ for tablet cured during $24 \mathrm{~h}$ in an oven and of $0.03 \% \mathrm{v} / \mathrm{v}$ for $4 \mathrm{~h}$ dynamically cured tablet.

In addition, Fig. 4 represents 3D reconstructed $\mathrm{X} \mu \mathrm{CT}$ images from segmentation procedure, allowing a better visualization of the presence of micro pores within the analyzed coating volumes. Two different sights of coating layers are illustrated. From these representations, the decrease in coating porosity after curing was clearly revealed by comparing the internal coating structures of uncured and cured tablets. A higher coating porosity was indeed obtained for uncured tablets $(0.2 \% \mathrm{v} / \mathrm{v})$. Interestingly, static curing or dynamic curing led to similar film-coating porosities.

Therefore, the use of X $\mu \mathrm{CT}$ provided further information about the internal quality of tablets in terms of coating porosity. These results were directly correlated to drug release properties previously described. The efficiency of a $4 \mathrm{~h}$ dynamic curing period was thus demonstrated by combining dissolution tests and X $\mu \mathrm{CT}$ measurements.

\subsection{Comparison of NIR and Raman spectral information}

NIR analysis of dynamically cured tablets recently revealed variations in absorbance in two NIR regions attributed to water. Indeed, the removal of water initially trapped within the coating layer was efficiently monitored along the dynamic curing operation by NIR spectroscopy (Gendre et al., 2012). Spectral evolution was observed with respect to dynamic curing times until $4 \mathrm{~h}$ by performing a principal component analysis from the pre-processed NIR spectra.

In this study, samples subjected to a $24 \mathrm{~h}$ static curing step were analyzed and compared to dynamically cured tablets. The two NIR regions over the spectral ranges $5060-5380 \mathrm{~cm}^{-1}$ and $6970-7190 \mathrm{~cm}^{-1}$, attributed to absorption bands of water, were first selected. Principal component analysis from NIR spectra highlighted two distinct behaviors depending on dynamic curing time and curing mode. Interestingly, samples corresponding to $24 \mathrm{~h}$ of static curing and at least to $4 \mathrm{~h}$ of dynamic curing were grouped in the same area (Fig. 5). Consequently based on these NIR results, curing carried out either in static conditions for $24 \mathrm{~h}$ or in dynamic conditions for at least $4 \mathrm{~h}$ led to similar NIR spectral information, which could be related to the water content of the coating layer. Interestingly, these observations corroborated dissolution and $\mathrm{X} \mu \mathrm{CT}$ results, which also demonstrated analogous film-coating structures.

In addition, Raman spectra analysis previously showed that the intensity of a specific Raman band at $1080 \mathrm{~cm}^{-1}$ decreased as dynamic curing proceeded, to entirely disappear after $4 \mathrm{~h}$. This result was attributed to a decrease in the gauche conformers along the alkyl chains of polymer, resulting in a denser and more organized coating structure.

To evaluate the influence of curing conditions on Raman information, samples cured in an oven for $24 \mathrm{~h}$ were analyzed. Similar

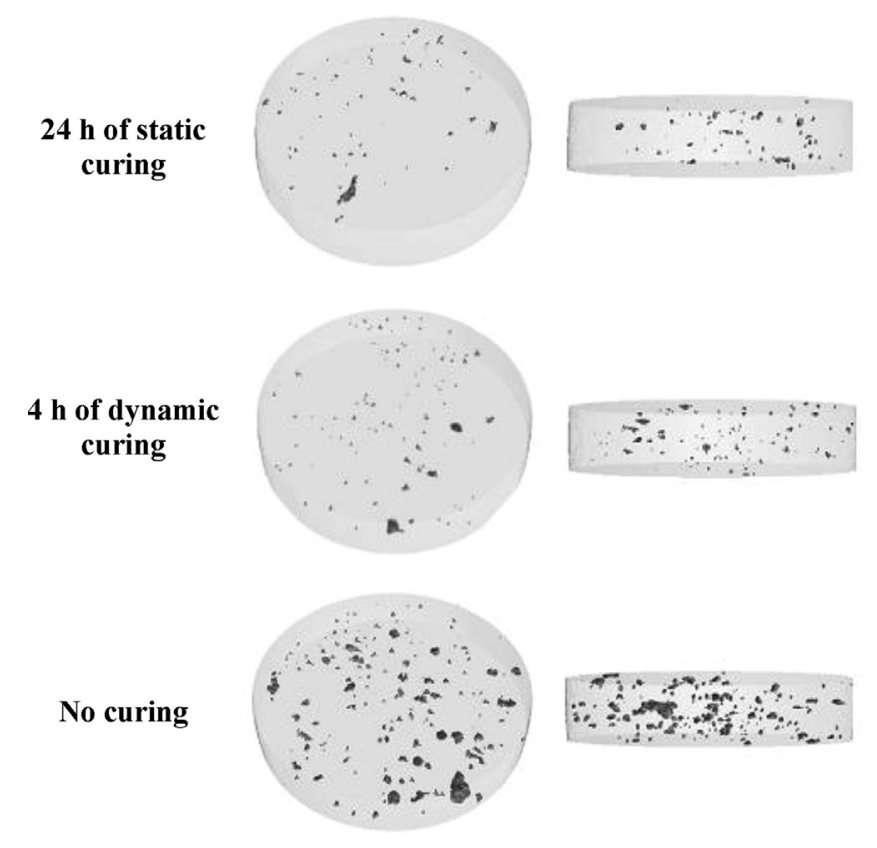

Fig. 4. 3D reconstructed X-ray micro-computed tomographic images of film-coating structures from tablet cured in static conditions for $24 \mathrm{~h}$ and from $4 \mathrm{~h}$ dynamically cured tablet. For comparison, film-coating structure from uncured tablet is also shown (selected cylindrical volume of $575 \mu \mathrm{m}$ diameter and of $56 \mu \mathrm{m}$ height). 


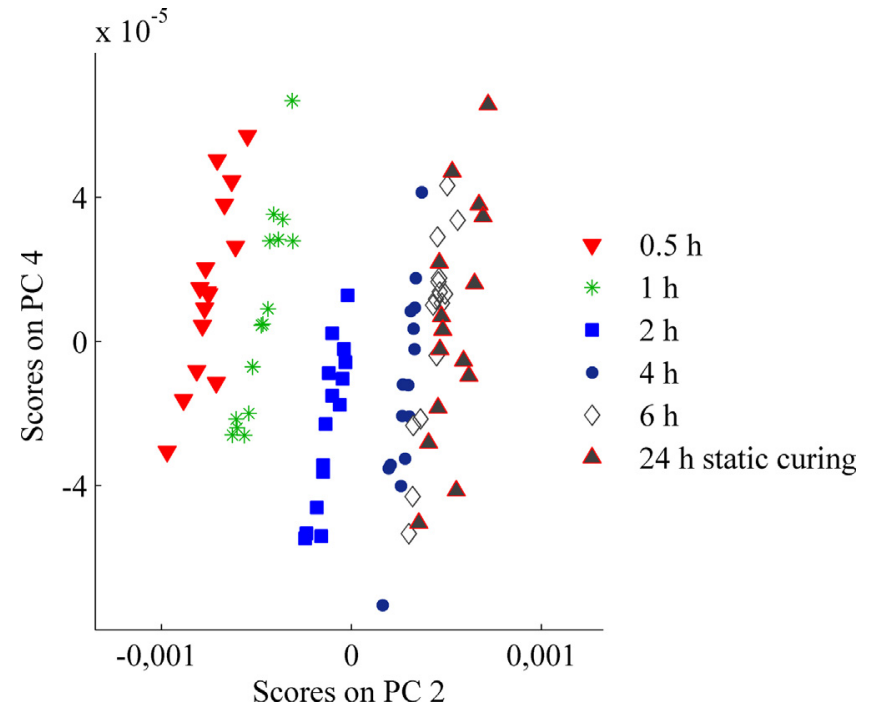

Fig. 5. PCA scores plot obtained from the pre-processed NIR spectra of coated tablets subjected either to $24 \mathrm{~h}$ of static curing in an oven or to dynamic curing ranging from $0.5 \mathrm{~h}$ to $6 \mathrm{~h}$ (results from dynamically cured tablets are reproduced from Gendre et al., 2012).

spectral features were obtained in comparison to the Raman spectra acquired from tablets after $4 \mathrm{~h}$ of dynamic curing. The disappearance of the $1080 \mathrm{~cm}^{-1}$ band confirmed the structural rearrangement of polymer chains during static curing, leading to a densification and a better organization of the coating layer.

Therefore, from these two complementary spectroscopic measurements, it clearly appeared that a stabilized state was reached after $24 \mathrm{~h}$ of static curing and $4 \mathrm{~h}$ of dynamic curing. Raman and near infrared techniques allowed the detection of slight variations within the coating layer which were directly correlated to dissolution results and image analysis of X $\mu \mathrm{CT}$ experiments. The use of these different characterization techniques can therefore provide a deeper insight and a better understanding of the underlying mechanisms associated with the curing process.

\subsection{Comparison of crystalline component distributions within the coating layer using $X$-ray microdiffraction}

Recent X-ray microdiffraction measurements performed on $4 \mathrm{~h}$ dynamically cured tablets revealed that the coating layer was in fact divided into an inner coating zone $(\sim 70 \mu \mathrm{m})$ and an outer coating zone $(\sim 30 \mu \mathrm{m})$ with different compositions, resulting in different diffraction patterns (Gendre et al., 2012). The presently used coating suspension contains partly crystallized ethylcellulose and two crystalline excipients, which are cetyl alcohol and sodium lauryl sulfate, incorporated in the commercially available aqueous ethylcellulose dispersion. Comparing X-ray microdiffraction patterns from two of these pure crystalline components and tablet cured under dynamic conditions for $4 \mathrm{~h}$, it was observed that the concentration of cetyl alcohol (presenting two intense WAXS peaks at $4.15 \AA$ and $3.68 \AA$ ) was higher in the external coating zone, decreasing rapidly until vanishing after a few tens of micrometers inside the coating. Concomitantly, the concentration of sodium lauryl sulfate (presenting two intense WAXS peaks at $4.32 \AA$ and $4.10 \AA$ ) was low in the external coating zone, increasing rapidly up to a few tens of micrometers inside the coating.

To confirm the crystalline component distributions within the coating structure and to study the influence of curing conditions, tablet cured in static conditions during $24 \mathrm{~h}$ was analyzed. Fig. 6 $\mathrm{A} 1$ and $\mathrm{B} 1$ show the resulting diffraction patterns. It can be noticed that the crystalline parameters in the lamellar plane were nearly the same to those previously obtained from $4 \mathrm{~h}$ dynamically cured

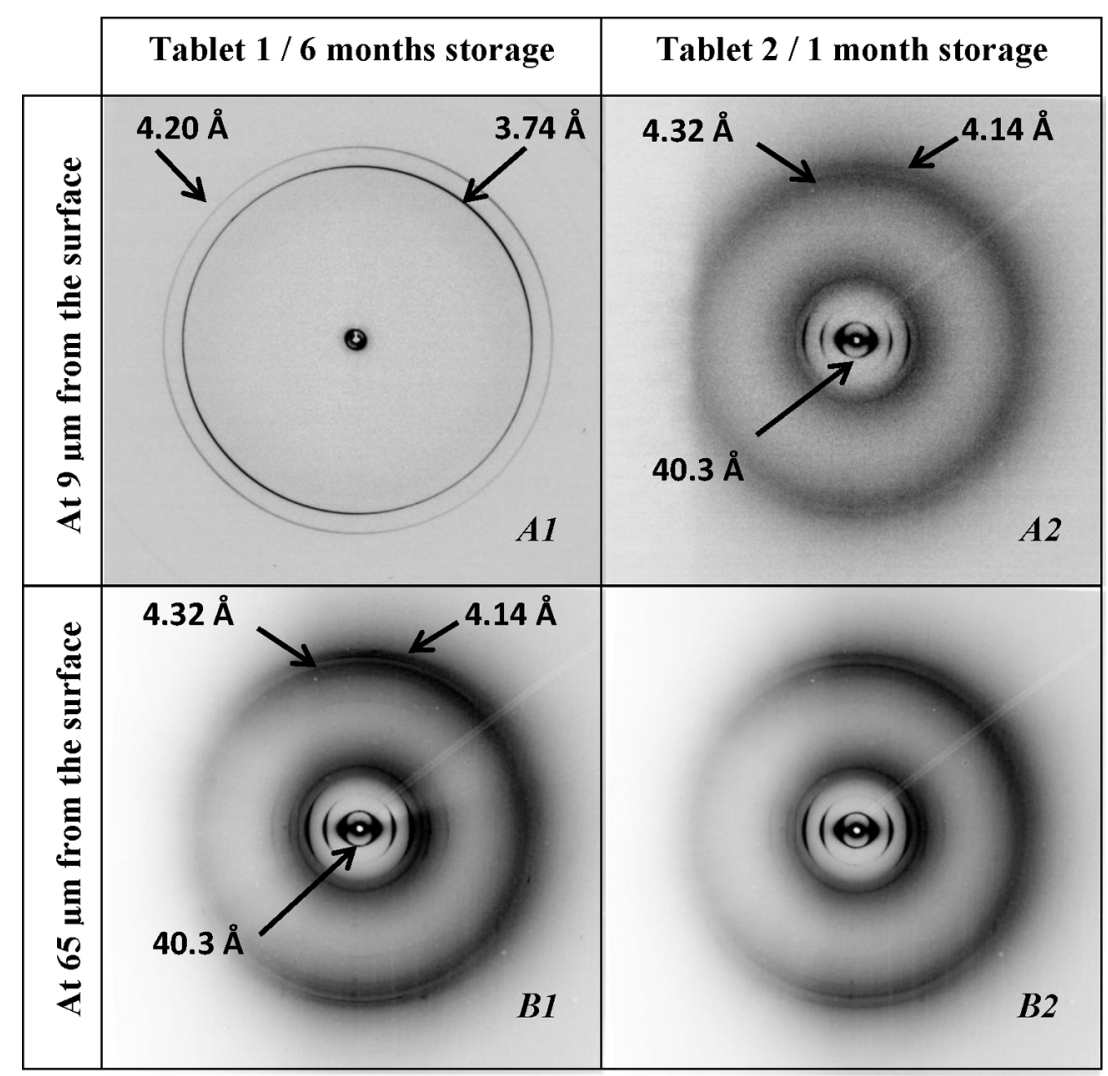

Fig. 6. X-ray microdiffraction patterns corresponding to tablet 1 stored for 6 months (left, patterns A1 and B1) and to tablet 2 stored for 1 month (right, patterns A2 and B2). 
tablets (intense WAXS peaks at $3.74 \AA$ and $4.20 \AA$ for cetyl alcohol and at $4.32 \AA$ and $4.14 \AA$ for sodium lauryl sulfate).

This last characterization technique completed the physicochemical determination of film-coating structures obtained after a curing step. All techniques demonstrated that $24 \mathrm{~h}$ of static curing and $4 \mathrm{~h}$ of dynamic curing resulted in similar film-coating properties.

\subsection{Detection of an unforeseen aging mechanism from X-ray microdiffraction measurements}

X-ray microdiffraction experiments were performed on two tablets cured in static conditions during $24 \mathrm{~h}$ but presenting distinct storages times. The above-described diffraction patterns were obtained from tablet 1 , stored 6 months at ambient conditions $\left(25^{\circ} \mathrm{C}\right.$ and $\left.60 \% \mathrm{RH}\right)$ (Fig. $6 \mathrm{~A} 1$ and $\mathrm{B} 1$ ). Importantly, the storage conditions ( 6 months storage) were similar for tablet 1 ( $24 \mathrm{~h}$ static curing) and for dynamically cured samples.

Surprisingly, the internal coating structure of tablet 2, stored 1 month at ambient conditions $\left(25^{\circ} \mathrm{C}\right.$ and $\left.60 \% \mathrm{RH}\right)$, was different (Fig. 6 A2 and B2). No external coating zone, previously attributed to crystalline cetyl alcohol, was detected. The crystalline coating distribution was homogeneous across the whole coating layer, corresponding to a uniform presence of crystalline sodium lauryl sulfate. Indeed, the crystalline coating structure of tablet 2 was found to be similar to the inner coating zone of tablet 1 . Therefore, it was assumed from this X-ray microdiffraction measurement that cetyl alcohol was in the amorphous state within the coating layer, in contrast to previous observations.

Taking into account the fact that the microdiffraction analyses were carried out after different storage times ( 6 months and 1 month), the formation of the outer coating zone may be attributed to an aging mechanism consisting in the diffusion and crystallization of the cetyl alcohol toward the surface of the coating. In addition, a downward migration of crystalline sodium lauryl sulfate may occur with time.

Therefore, the use of X-ray microdiffraction revealed excipient crystallization and possible migration phenomena based on tablet storage conditions. Nevertheless, no direct correlation with the curing process can be established. Changes in drug crystallinity occurring with heat treatment were described in the case of watersoluble drugs and attributed to drug migration into the coating layer (Nikowitz et al., 2013). Drug release properties can consequently be modified due to interaction between core ingredients and coating formulation (Sadeghi et al., 2003). In our case, further quantitative analyses would have to be performed to elucidate $\mathrm{X}$ ray microdiffraction observations and to study their influences on coating properties.

\section{Conclusions}

Influence of static curing versus dynamic curing on filmcoating structures was widely characterized. Various advanced characterization techniques were investigated and allowed deeper physico-chemical insights into cured coating layers. Image analysis of X $\mu \mathrm{CT}$, NIR and Raman spectroscopic measurements and $\mathrm{X}$-ray microdiffraction experiments revealed similar film-coating structures either after $24 \mathrm{~h}$ of traditional oven curing or after $4 \mathrm{~h}$ of dynamic curing. These results confirmed the equivalent drug release properties obtained for the two curing conditions. Consequently, the relevant determination of the dynamic curing end-point was validated. The efficiency of dynamic curing can foresee increased development of industrial curing applications.

Furthermore, X-ray microdiffraction analysis pointed out a surprising aging mechanism depending on storage time which may be related to the crystallization and the upward migration of cetyl alcohol associated to the downward migration of crystalline sodium lauryl sulfate during storage.

\section{Acknowledgements}

Dr. Barbara Fayard (Novitom), Dr. Jean Doucet (Novitom) and Dr. Michel Manfait (MéDIAN Unit, CNRS UMR 6237, Faculty of Pharmacy, Reims Champagne-Ardenne University, France) are acknowledged for $\mathrm{X} \mu \mathrm{CT}$ experiments, for X-ray microdiffraction measurements and for Raman facilities, respectively. We thank the Brazilian Bioscience National Laboratory (LNBio) for financial support of J.C. da Silva. Claire Gendre was supported by a CIFRE fellowship granted by Technologie Servier and the French Ministry of Research and Innovation.

\section{References}

Baer, H., Weibord, W., Peterit, H.U., Skalsky, B., 2008. Improved Curing of Eudragit ${ }^{\circledR}$ RL/RS 30D Film Coatings by Controlled In-process Curing in the Fluid Bed. Poster Communication, 6th World Meeting of Pharmaceutics, Biopharmaceutics and Pharmaceutical Technology, APV, Barcelona.

Bodmeier, R., Paeratakul, O., 1994. The effect of curing on drug release and morphological properties of ethylcellullose pseudolatex-coated beads. Drug. Dev. Ind. Pharm. 20, 1517-1533.

Felton, L.A., 2013. Mechanisms of polymeric film formation. Int. J. Pharm., http://dx.doi.org/10.1016/j.ijpharm.2012.12.027.

Gendre, C., Genty, M., da Silva, J.C., Tfayli, A., Boiret, M., Lecoq, O., Baron, M., Chaminade, P., Péan, J.M., 2012. Comprehensive study of dynamic curing effect on tablet coating structure. Eur. J. Pharm. Biopharm. 81, 657-665.

Körber, M., Hoffart, V., Walther, M., Macrae, R.J., Bodmeier, R., 2010. Effect of unconventional curing conditions and storage on pellets coated with Aquacoat ECD. Drug. Dev. Ind. Pharm. 36, 190-199.

Liu, J., Williams III, O.R., 2002. Properties of heat-humidity cured cellulose acetate phtalate free films. Eur. J. Pharm. Sci. 17, 31-41.

Monkare, J., Pajander, J., Hakala, R.A., Savolainen, P., Jarvelainen, M., Korhonon, H., Seppala, J.V., Jarvinen, K., 2012. Characterization of internal structure polymer erosion and drug release mechanisms of biodegradable poly(ester anhydride)s by X-ray microtomography. Eur. J. Pharm. Sci. 47, 170-178.

Moore, J.W., Flanner, H.H., 1996. Mathematical comparison of dissolution profiles. Pharm. Technol. 20, 64-74.

Muschert, S., Siepmann, F., Leclercq, B., Siepmann, J., 2011. Dynamic and static curing of ethylcellulose: PVA-PEG graft copolymer film coating. Eur. J. Pharm. Biopharm. 78, 455-461.

Naes, T., Isaksson, T., Fearn, T., Davies, T., 2002. A User-friendly Guide to Multivariate Calibration and Classification. NIR Publications, Chichester.

Nikowitz, K., Pintye-Hodi, K., Regdon Jr., G., 2013. Study of the recrystallization in coated pellets - effects of coating on API crystallinity. Eur. J. Pharm. Sci. 48, 563-571

Noguchi, S., Kajihara, R., Iwao, Y., Fujinami, Y., Suzuki, Y., Terada, Y., Uesugi, K., Miura, K., Itai, S., 2013. Investigation of internal structure of fine granules by microtomography using synchrotron X-ray radiation. Int. J. Pharm. 445, 93-98.

Porter, S., Sackett, G., Liu, L., 2009. Development, optimization and scale-up of process parameters: pan coating. In: Qiu, Y., Chen, Y., Zhang, G.G.Z. (Eds.), Developing Solid Oral Dosage Forms: Pharmaceutical Theory and Practice. , 1st ed. Academic Press, New York, pp. 761-805.

Poutiainen, S., Pajander, J., Savolainen, A., Ketolainen, J., Jarvinen, K., 2011. Evolution of granule structure and drug content during fluidized bed granulation by X-ray microtomography and confocal Raman spectroscopy. J. Pharm. Sci. 12, 5254-5269.

Sadeghi, F., Ford, J.L., Rajabi-Siahboomi, A., 2003. The influence of drug type on the release.profiles from Surelease-coated pellets. Int. J. Pharm. 254, 123-135.

Siepmann, F., Hoffmann, A., Leclercq, B., Carlin, B., Siepmann, J., 2007. How to adjust desired drug release patterns from ethylcellulose-coated dosage forms. J. Control. Release 119, 182-189.

Wesseling, M., Bodmeier, R., 1999. Drug release from beads coated with an aqueous colloidal ethylcellulose dispersion Aquacoat, or an organic ethylcellulose solution. Eur. J. Pharm. Biopharm. 47, 33-38. 